\title{
Autoconcepto del adolescente según sus características sociodemográficas y de los progenitores
}

\author{
Adolescent self-concept according to their sociodemographic and parental characteristics
}

\author{
Juana Morales-Quizhpi iD , María-Dolores Palacios-Madero iD, Elsa Conforme-Zambrano iD, \\ Nube Arpi-Peñaloza iD \\ Proyecto "Apoyo-control parental y promoción de la autonomía, del autoconcepto y autorregulación del aprendizaje en \\ adolescentes de Cuenca" (Referencia Proyecto:2040000071632), Universidad de Cuenca, Cuenca, Ecuador. \\ Fecha de recepción: 31 de marzo de 2021 - Fecha de aceptación: 23 de abril de 2021
}

\begin{abstract}
RESUMEN
La adolescencia implica cambios psicosociales importantes, uno de ellos, es el desarrollo del autoconcepto que se afianza en esta etapa en un contexto familiar, académico y social. El objetivo del estudio fue analizar las dimensiones del autoconcepto de los adolescentes cuencanos, en función de: i) las características sociodemográficas: sexo, escolaridad, sostenimiento institucional y la tipología familiar de los adolescentes, ii) las características sociodemográficas de los progenitores: jornada laboral, el estado civil y el nivel de estudios. Participaron 1085 adolescentes escolarizados entre 15 y 18 años de la ciudad de Cuenca, se aplicó la Escala Autoconcepto Forma 5 (García \& Musitu, 2014). Los resultados informan que los adolescentes cuencanos presentan puntuaciones por encima del percentil 50 en la dimensión académica y física; seguidas por la dimensión familiar y emocional; mientras que, la dimensión social reporta una puntuación ligeramente por debajo del percentil 50. En cuanto al sexo, los hombres presentan una puntuación superior en la dimensión social y familiar en comparación a las mujeres, quienes presentan un mejor autoconcepto académico, emocional y físico. Según la escolaridad, los adolescentes de tercer año de bachillerato muestran un autoconcepto físico y los que asisten a instituciones particulares un mejor autoconcepto académico $y$ familiar, al igual que los adolescentes cuyos padres y madres tienen un estado civil de casado y un nivel de estudios de posgrado. Se concluye que la variabilidad de las dimensiones del autoconcepto del adolescente se explica por la presencia de las características sociodemográficas de los adolescentes y de sus progenitores.
\end{abstract}

Palabras clave: Autoconcepto, adolescentes, características sociodemográficas, progenitores.

\begin{abstract}
Adolescence implies important psychosocial changes, one of them is the development of the self-concept that takes hold at this stage in a familiar, academic, and social context. The objective of the study was to analyze the dimensions of the selfconcept of adolescents from Cuenca, based on: i) the sociodemographic characteristics: sex, schooling, institutional support and the family typology of the adolescents, ii) the sociodemographic characteristics of the parents: working hours, marital status and educational level. 1085 schooling adolescents between 15 and 18 years old from the city of Cuenca participated, the Self-Concept Scale Form 5 was applied (García \& Musitu, 2014). The results reported that Cuenca adolescents present scores above the 50th percentile in the academic and physical dimension; followed by the family and emotional dimension, while the social dimension reports a score slightly below the 50th percentile. Regarding gender, men present a higher score in the social and familiar dimension compared to women, who present a better academic, emotional, and physical self-concept. According to schooling, adolescents in the third year of high school show a physical self-concept and those who attend private institutions a better academic and family self-concept, as do adolescents whose parents have a married marital status and a postgraduate level of studies. In conclusion variability of the dimensions of adolescent self-concept is explained by the presence of the sociodemographic characteristics of adolescents and their parents.
\end{abstract}

Keywords: Self-concept, adolescents, sociodemographic characteristics, parents.

\section{INTRODUCTION}

Estudiar el autoconcepto es particularmente importante en la adolescencia, al ser una etapa marcada por continuos cambios biológicos y psicológicos, así como de aprendizajes y oportunidades, una etapa donde el adolescente asume la formación de su propia identidad
(Erikson, 1992; Fuentes et al., 2015; Oliva et al., 2014; Suldo et al., 2011). En la adolescencia, uno de los elementos estrechamente relacionado con la adaptación psicológica y social es el autoconcepto (Delgado et al., 2013; Fuentes et al., 2011; Shavelson et al., 1976).

El autoconcepto puede ser definido como "un conjunto de autopercepciones de sí mismo que se forman a través de 
las experiencias con el entorno y las interpretaciones de dichas experiencias, las cuales tienen una fuerte influencia de los reforzamientos y evaluaciones de otras personas significativas" (Shunk, 2012, p. 490); además, se considera como la imagen o percepción descriptiva que uno tiene de sí mismo como un juicio reflexivo acerca de las propias capacidades, valores, preferencias $\mathrm{o}$ apariencias (García, 2002; Heathertorn \& Wyland, 2003), considerado como un proceso cognitivo con incidencia en el comportamiento de las personas (Garaigordobil \& Durá, 2006).

Al ser el autoconcepto un predictor de la conducta, permite que los individuos que se sientan competentes actúen con mayor determinación y seguridad ante diferentes tareas (Fuentes et al., 2011). En esta dirección, los adolescentes con un autoconcepto positivo se mostrarán estables frente a las demandas presentadas por el entorno, con bajas puntuaciones en escalas de depresión $\mathrm{y}$ ansiedad, se vuelven menos propensos a sufrir trastornos alimenticios, y muestran una mejor integración social en sus aulas (Fuentes et al., 2015). Así también, diferentes estudios relacionan un alto autoconcepto familiar con mejores resultados de la socialización familiar en los hijos. Adicionalmente, se encontró que los adolescentes con alto autoconcepto académico muestran un mayor logro escolar y rendimiento académico, y los que presentan un alto autoconcepto emocional presentan buena estabilidad emocional. De estos resultados se infiere que la persona se acerca a un mejor ajuste psicosocial (García, 2002; García \& Román, 2005; De La Torre et al., 2013; Esnaola et al., 2008; Goñi \& Fernández, 2008; Ros \& Zuagagoitia, 2015). Por el contrario, cuando en la adolescencia se presenta un autoconcepto negativo disminuye el sentido de identidad propia, siendo más fácil caer en depresión, consumo de sustancias, alcohol, mala actitud hacia las actividades escolares, dificultades en la socialización, menospreciarse, rechazar cumplidos, burlarse de los demás, mostrar una cantidad excesiva de enojo (Esnaola et al., 2008; Gasa Molepo \& Lethole, 2018; Fuentes et al., 2011; Moreno et al., 2017).

Al investigar la variable de autoconcepto se señala que existen dos formas de abordar este constructo teórico; una perspectiva unidimensional y otra multidimensional y jerárquica. Desde esta última perspectiva la realidad multidimensional y jerárquica del autoconcepto es entendida como las diversas dimensiones del autoconcepto interrelacionadas, pero al mismo tiempo diferentes, el modelo plantea que el autoconcepto del adolescente puede dividirse en autoconcepto académico y autoconcepto no-académico, configurándose este último por componentes emocionales que son los más subjetivos e internos; los sociales están relacionados con el significado que la conducta del individuo tiene para los demás, mientras que los físicos tienen una incidencia fundamental las actitudes y apariencia general del individuo (Garcia \& Musitu, 2014).

Desde esta mirada, el autoconcepto integra las dimensiones: académica, física, emocional, familiar y social, que explica las diferentes autoconcepciones que la persona manifiesta en los diferentes ámbitos de su vida familiar, social, académica escolar o comunitaria. Por otra parte, una de las características más sorprendentes y discutidas junto con la de la multidimensionalidad del autoconcepto es su capacidad de permanecer estable y cambiante al mismo tiempo; de ser estable en sus aspectos más nucleares y profundos, a la vez que variable en sus aspectos más dependientes del contexto. Mientras que, la naturaleza jerárquica de sus dimensiones tienen diferentes significados e importancia en función de los valores y de la edad de los sujetos, lo que explica la presencia de una dimensión más general y estable que se sitúa sobre otras más específicas en niveles intermedios e inferiores (Esnaola et al., 2011; Mérida et al., 2015; García et al., 2018).

Desde esta perspectiva describimos las dimensiones del autoconcepto, las mismas que representan diferentes cualidades que se relacionan diferencialmente con distintas áreas del comportamiento humano: 1) el autoconcepto académico se refiere a la percepción que los sujetos tienen de la calidad de su desempeño como estudiante; 2) el autoconcepto social, que refleja las percepciones que los sujetos tienen de su desempeño en las relaciones sociales; 3 ) el autoconcepto emocional, que captura la percepción del propio estado emocional del individuo y las respuestas a situaciones concretas; 4) el autoconcepto familiar, que refleja la percepción del sujeto sobre su implicación, participación e integración en el ámbito familiar, y; 5) el autoconcepto físico, percepción que la persona tiene de su apariencia y desempeño físico (Inda-Caro et al., 2020; García et al., 2018).

La perspectiva multidimensional considera la influencia de diferentes ámbitos en los que interactúa el adolescente, resaltando entre ellos, la percepción de los progenitores que constituye la influencia más significativa, hasta el punto de determinar en gran parte su autoconcepto (Esnaola et al., 2011). Adicionalmente, diferentes investigaciones ponen de manifiesto el efecto de la familia sobre el autoconcepto, siendo los adolescentes con niveles elevados de autoconcepto y adaptación personal aquellos que perciben su ambiente familiar más cohesionado y poco conflictivo (De La Torre et al., 2013; GonzálezPienda, et al., 2002; Pichardo \& Amezcua, 2001; Putnick, et al., 2008).

Se debe considerar que las prácticas parentales, sus creencias y expectativas acerca de la capacidad y logros del adolescente, favorecen el desarrollo de un autoconcepto académico, factores que determinan los resultados académicos de los adolescentes (Rodríguez \& Ramos 2015; Ros \& Zuagagoitia, 2015). Es así como, referente al autoconcepto y logros académicos, estos dependen principalmente de la situación social, económica y educativa de la familia. Al respecto Rodrigo \& Palacios (1998) postulan que las prácticas parentales se relacionan con una serie de factores que se dividen en tres grupos: el primero relacionado con los hijos, un segundo relativo a los padres; $y$, un tercer factor relacionado con la situación o contexto en que se presenta la interacción.

Sobre las características de los adolescentes, investigaciones explican la variabilidad del autoconcepto según la edad y el sexo de los adolescentes. En esta dirección Esnaola et al. (2011) postulan que el autoconcepto aumenta su multidimensionalidad con la edad. Estudios encontraron que el autoconcepto académico presenta puntuaciones más altas en adolescentes de mayor edad que permite una mayor diferenciación interna y una mayor distinción con respecto a otras dimensiones (Esnaola, 2005, 2011; Rodríguez \& Ramos, 2017). En lo referente a la variabilidad del autoconcepto según el sexo Derdikman-Eiron et al. (2011) señalan que el autoconcepto general es favorable en los hombres. En tanto que las variaciones de mayor magnitud 
de las dimensiones de autoconcepto no académicas son favorables a las mujeres (Tomyn \& Cummins, 2011).

En cuanto a la tipología familiar del adolescente, estudios indican que, si bien la configuración familiar no parece ser la clave del mejor o peor ajuste emocional y psicosocial de los hijos, ciertas tipologías familiares como las monoparentales tienen más probabilidad para el ajuste psicosocial. Sin embargo, los hijos pueden tener un buen desarrollo del autoconcepto y ajuste psicosocial en cualquier estructura familiar, si se cumplen ciertas condiciones básicas necesarias para el bienestar de sus miembros y pueden mantener vínculos sociales a través de la vida familiar (Oliva et al., 2014; Oudhof et al., 2018; Martínez et al., 2013; Viveros, 2016). A pesar de ello, no podemos dejar de considerar que actualmente la disminución de matrimonios, altas tasas de separaciones y divorcios han generado una modificación del estado civil de los progenitores y una creciente diversificación en cuanto a la conformación de los tipos de familia, con las cuales se rompe la hegemonía de la familia nuclear o tradicional (Castaño et al., 2020).

Respecto a las características de los progenitores resaltamos el nivel educativo, indican que la educación de los padres ayuda a los adolescentes a desarrollar características de personalidad adecuadas y los prepara para adaptarse social y académicamente (Morsy \& Rothstein, 2015). Se cree que un alto nivel de estudios de los padres conduce a un patrón de comportamiento más positivo y saludable en los adolescentes. Influye en sus creencias, comportamientos y expectativas, lo que conduce a resultados positivos en el autoconcepto académico y el rendimiento escolar (Gasa et al., 2018).

Finalmente, mencionamos a la jornada laboral de los progenitores como otro factor influyente con las dimensiones del autoconcepto, al respecto Mistry et al. (2007) manifestaron que acontecimientos estresantes como elevadas jornadas de trabajo, bajo nivel económico y desempleo desencadenarían tensiones en el clima familiar y estarían relacionados con las prácticas en la crianza de la progenie. En esta línea Cleric et al. (2020) mencionan que el estatus socioeconómico juega un papel importante en la determinación del nivel de autoconcepto en un individuo. Es así como diversos entornos socioeconómicos también generarían un impacto en el autoconcepto de los adolescentes, en dimensiones tales como: la autopercepción corporal, el rendimiento académico y la autopercepción familiar y social. Las autoras del estudio consideran en esta investigación que los factores de la crianza como las características de los adolescentes y de los progenitores, podrían ser mediadores de las dimensiones del autoconcepto.

La familia es el principal referente en la construcción del autoconcepto en una de las etapas vitales como es la adolescencia, a pesar de ello, existen pocas investigaciones a nivel nacional y local que informen de la relación de las características sociodemográficas de los adolescentes y progenitores en el desarrollo del autoconcepto. De acuerdo con la situación descrita, esta investigación tuvo como propósito analizar las dimensiones del autoconcepto de los adolescentes cuencanos, en función de: i) las características sociodemográficas de sexo, escolaridad, sostenimiento institucional y tipología familiar de los adolescentes, ii) las características sociodemográficas de los progenitores: la jornada laboral, el estado civil y el nivel de estudios.

\section{MATERIALES Y MÉTODO}

La investigación asume una metodología cuantitativa, un diseño descriptivo relacional de corte transversal y no experimental, considerando que la recolección de datos se realizó de una manera estructurada y secuencial en un periodo determinado y una población de estudio definida. El tamaño de la muestra se calculó con la base de datos del Archivo Maestro de Instituciones Educativas (AMIE), del periodo lectivo 2018-2019 con un nivel de confianza del $95 \%$ y $3 \%$ de error.

Los resultados reportaron que el tamaño muestral mínimo necesario era de 1029 estudiantes. Para la selección de instituciones educativas, se empleó un método polietápico; fijando un número óptimo de 18 instituciones para una operatividad en la recolección de información eficiente; posteriormente la selección de los paralelos de primero, segundo y tercero año de bachillerato se realizó de forma aleatoria. Contemplando los procedimientos se solicitó la autorización de la Coordinación Zonal 6 de Educación, y de los directivos de las 18 instituciones educativas; posteriormente se procedió con la recolección de la información. En la ejecución de la investigación se consideró los aspectos éticos propuestos por el Comité de Bioética del Área de la Salud (COBIAS), que regula a los proyectos de investigación en la Universidad de Cuenca, garantizando la confidencialidad en el manejo de la información, y responsabilidad de cumplir y hacer cumplir con las normas bioéticas nacionales e internacionales durante todo el proceso de desarrollo del trabajo. Por medio de consentimientos y asentimientos que de manera previa fueron autorizados por los progenitores de los estudiantes que formaron parte de la investigación. La aplicación de los instrumentos tuvo una duración aproximada de 20 minutos.

Se aplicó el instrumento de investigación a estudiantes: 506 mujeres y 579 hombres, con una escolaridad de primer año $(\mathrm{n}=35.4 \%, \mathrm{M}=15.2$ años; $\mathrm{DE}=0.8)$, segundo año $(\mathrm{n}=34.1 \%, \mathrm{M}=16.2 ; \mathrm{DE}=0.7)$ y tercer año $(\mathrm{n}=30.5 \%$; $\mathrm{M}=17.15$; $\mathrm{DE}=0.8$ ) de Bachillerato General Unificado (BGU); de instituciones educativas de sostenimiento público $(41.5 \%)$, particular $(50.7 \%)$ y fiscomisional (7.8\%). El 51.9\% de estudiantes pertenecían a una familia nuclear siendo la tipología de familia predominante, seguida por la monoparental materna $(19.7 \%)$ y la familia extendida $(18.1 \%)$; además el $8.5 \%$ pertenecía a una familia reconstituida y el $1.96 \%$ restante correspondía a una familia monoparental paterna. Esta información se recolectó a través de una ficha sociodemográfica para la caracterización de la población de estudio, en cuanto a sexo, edad, el año de escolaridad, la tipología familiar.

Para la identificación de las dimensiones del autoconcepto de los adolescentes se utilizó la Escala Autoconcepto Forma 5 (AF5) (García \& Musitu, 2014), está compuesta por 30 ítems que evalúan 5 dimensiones del autoconcepto: académico "Hago bien mi tarea", social "Soy una persona amigable", emocional con ítems invertidos "Muchas cosas me ponen nervioso", familia "Siento que mis padres me aman" y físico "Me gusta cómo me veo". La escala tiene 6 ítems por dimensión con una escala de respuesta que oscila de 1 (totalmente en desacuerdo) a 99 (totalmente de acuerdo). La confiabilidad del instrumento se determinó con el del análisis de consistencia interna, alfa de Cronbach, con una confiabilidad global de $\alpha=.815$. 
El análisis de los datos se realizó en el programa estadístico SPSS 25. Los resultados generales de la escala aplicada se muestran mediante medidas de tendencia central y dispersión, debido a que los datos no siguen una distribución normal se aplicó pruebas no paramétricas: la prueba U Mann Whitney para la comparación entre dos grupos, y Kruskal y Wallis para más de dos grupos (autoconcepto, características familiares de progenitores y tipología familiar de los adolescentes); se empleó el coeficiente de correlación rho de Spearman, todas con una significancia del 5\%.

\section{RESULTADOS}

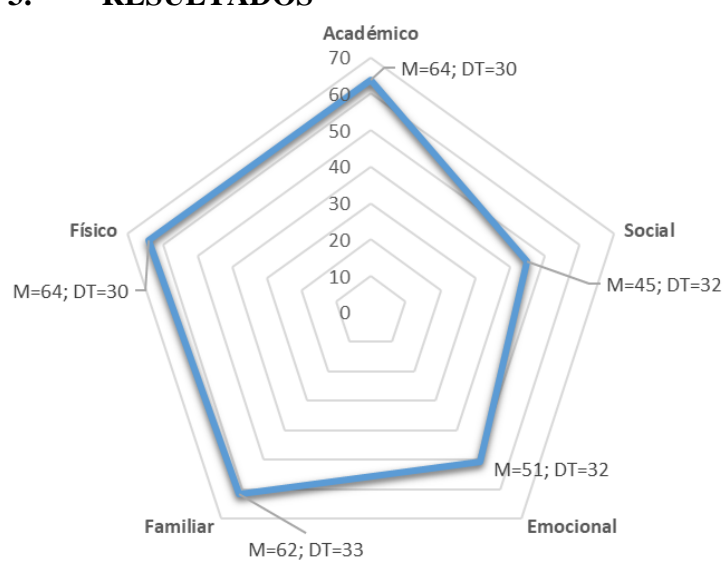

Figura 1. Dimensiones del autoconcepto en los adolescentes cuencanos. Basado en el punto de la distribución percentil 50 como punto de corte.

Del análisis descriptivo se informa que las dimensiones del autoconcepto en los adolescentes cuencanos son altas considerando que el punto de la distribución es el percentil 50, siendo más alta la dimensión académica y física con igual puntuación $(\mathrm{M}=64 ; \mathrm{DT}=30)$; seguida por la dimensión familiar $(\mathrm{M}=62 ; \quad \mathrm{DT}=33)$ y dimensión emocional $(\mathrm{M}=51$; $\mathrm{DT}=33)$, mientras que la dimensión social $(\mathrm{M}=45$; $\mathrm{DT}=32)$ presentó una puntuación más baja. En cada una de las dimensiones se registraron altas dispersiones de datos lo que implica un comportamiento heterogéneo (Fig. 1).

\section{Dimensiones del Autoconcepto de los adolescentes según sus características sociodemográficas}

A partir de la Tabla 1, se analiza las dimensiones del autoconcepto del adolescente en función de sus variables sociodemográficas. Se observa variabilidad en las dimensiones considerando el sexo de los adolescentes, es así que, los hombres y mujeres presentan puntuaciones por encima de la media en las dimensiones: académica (Mhombre $\left.=62 ; \quad \mathrm{DT}=29 ; \quad \mathrm{M}_{\text {mujer }}=66 ; \quad \mathrm{DT}=31\right)$, familiar $\left(M_{\text {hombre }}=66 * ; \quad D T=31 ; \quad M_{\text {mujer }}=56 ; \quad D T=34\right)$, y física $\left(M_{\text {hombre }}=63 ; \mathrm{DT}=31 ; \mathrm{M}_{\text {mujer }}=66 ; \mathrm{DT}=29\right)$, en tanto que la dimensión social se ubica por debajo de la media $\left(M_{\text {hombre }}=48 * ; \mathrm{DT}=32 ; \mathrm{M}_{\text {mujer }}=42 ; \mathrm{DT}=32\right)$. Respecto a la dimensión emocional, son las mujeres que presentan una puntuación ligeramente superior al punto de corte $(\mathrm{M}=54$; $\mathrm{DT}=32$ ), mientras que los hombres presentan un valor por debajo de la media $(\mathrm{M}=48$; $\mathrm{DT}=32)$.

En cuanto a las variables sociodemográficas: la escolaridad, el sostenimiento institucional y la tipología familiar de los adolescentes muestran un comportamiento similar, es decir, la dimensión académica, emocional, familiar y física se encuentran por encima de la media y la dimensión social se ubica por debajo de la media.

Al relacionar las dimensiones del autoconcepto con las variables sociodemográficas mencionadas se observó que la dimensión académica de los adolescentes cuencanos mostró una relación significativa con el sostenimiento, puntuando más alto la institución particular $\left(\mathrm{M}=71^{*}\right.$; $\mathrm{DT}=28$ ), por su parte, la dimensión social se asocia con la variable sexo, siendo los hombres los que presentan el promedio más alto $\left(\mathrm{M}=48^{*} ; \mathrm{DT}=32\right)$. Por otro lado, la dimensión física del autoconcepto se relaciona la escolaridad, destacando el tercer año de bachillerato el punto más alto $\left(M=68^{*}\right.$; DT=29), se puede enfatizar que la dimensión familiar presenta una asociación con la tipología familiar, siendo la variable familia nuclear la que presenta una mayor puntuación $(\mathrm{M}=64.3 *$; $\mathrm{DT}=32)$, con la sostenibilidad se identifica el punto más alto en la institución particular (M=66*; DT=32) y considerando la variable de sexo de los participantes, los hombres puntúan más alto $\left(\mathrm{M}=66^{*} ; \mathrm{DT}=31\right)$ y la dimensión emocional no reporta ninguna relación con las características sociodemográficas de los adolescentes.

Tabla 1. Autoconcepto según la característica sociodemográficas de los adolescentes.

\begin{tabular}{llcccccccccc}
\hline \multirow{2}{*}{ Característical } & & \multicolumn{3}{c}{ Académico } & \multicolumn{3}{c}{ Social } & \multicolumn{3}{c}{ Emocional } & \multicolumn{3}{c}{ Familiar } & \multicolumn{2}{c}{ Físico } \\
\cline { 2 - 11 } & & M & DT & M & DT & M & DT & M & DT & M & DT \\
\hline \multirow{2}{*}{ Sexo } & Mujer & 66 & 31 & 42 & 32 & 54 & 32 & 56 & 34 & 66 & 29 \\
& Hombre & 62 & 29 & $48^{*}$ & 32 & 48 & 32 & $66^{*}$ & 31 & 63 & 31 \\
\hline \multirow{2}{*}{ Curso de } & Primero & 64 & 30 & 45 & 32 & 52 & 33 & 62 & 33 & 61 & 31 \\
bachillerato & Segundo & 63 & 31 & 45 & 32 & 48 & 30 & 61 & 34 & 64 & 31 \\
& Tercero & 64 & 29 & 44 & 32 & 52 & 32 & 63 & 33 & $68^{*}$ & 29 \\
\hline \multirow{3}{*}{ Sostenimiento } & Pública & 57 & 31 & 42 & 32 & 50 & 32 & 58 & 34 & 63 & 33 \\
& Particular & $71^{*}$ & 28 & 47 & 32 & 52 & 32 & $66^{*}$ & 32 & 65 & 30 \\
& Fiscomisional & 65 & 27 & 39 & 31 & 51 & 32 & 58 & 33 & 63 & 31 \\
\hline \multirow{5}{*}{ Tipología de } & Nuclear & 66 & 29 & 46 & 32 & 51 & 31 & $64.3 *$ & 32 & 66 & 30 \\
familia & Extendida & 63 & 30 & 46 & 31 & 49 & 34 & 63 & 33 & 61 & 31 \\
& Reconstituida & 59 & 32 & 47 & 32 & 54 & 34 & 53 & 35 & 64 & 31 \\
& Monoparental materna & 62 & 30 & 41 & 32 & 51 & 31 & 59 & 34 & 62 & 31 \\
& Monoparental paterna & 58 & 32 & 56 & 27 & 62 & 33 & 50 & 35 & 71 & 35 \\
\hline
\end{tabular}

Nota: $*$ (p-valor $<0.05)$. 


\section{Dimensiones del autoconcepto de los adolescentes con relación a las características sociodemográficas de la madre}

El análisis de los resultados de la relación entre las dimensiones del autoconcepto y las características sociodemográficas de la madre se exponen en la Tabla 2. En cuanto a la jornada laboral se observa que los adolescentes cuencanos con madres que trabajan 8 o más horas presentan puntuaciones por encima del percentil 50 en la dimensión académica $(\mathrm{M}=65.5 ; \mathrm{DT}=29.3 ; \mathrm{M}=64.9$; $\mathrm{DT}=28.5 ; \mathrm{M}=67.5 ; \mathrm{DT}=32.8$ ), dimensión emocional $(\mathrm{M}=52.2 ; \quad \mathrm{DT}=32.2 ; \quad \mathrm{M}=59.8 ; \quad \mathrm{DT}=35.5), \quad$ familiar $(\mathrm{M}=61.5 ; \quad \mathrm{DT}=32.8 ; \quad \mathrm{M}=60,8 ; \quad \mathrm{DT}=31.1 ; \quad \mathrm{M}=56.4$; $\mathrm{DT}=40.4)$ y física $(\mathrm{M}=64.9 ; \mathrm{DT}=29.7 ; \mathrm{M}=65.3$; $\mathrm{DT}=29.8$; $\mathrm{M}=57.0$; DT=37.4). Así también, los adolescentes con madres que trabajan menos de 8 horas muestran una mayor puntuación por encima del percentil 50 en la dimensión académica $(\mathrm{M}=56.1 ; \quad \mathrm{DT}=32.8 ; \mathrm{M}=65.0$; $\mathrm{DT}=29.5), \quad$ familiar $\quad(\mathrm{M}=55.5 ; \quad \mathrm{DT}=34.9 ; \quad \mathrm{M}=63.3$; $\mathrm{DT}=33.3)$ y la dimensión física $(\mathrm{M}=64.1 ; \mathrm{DT}=28.0$; $\mathrm{M}=63.5 ; \mathrm{DT}=30.2$ ). Los resultados en la dimensión social se reportan por debajo del percentil 50 con respecto a la jornada de trabajo de la madre.

Respecto al cruce de la variable del estado civil de la madre y las dimensiones del autoconcepto, los resultados informan que los adolescentes cuencanos cuyo estado civil de la madre responde a soltera, casada o en unión libre, divorciada y viuda presentan puntuaciones sobre el percentil 50 en las dimensiones: académica, familiar y física. Los hallazgos evidencian que los adolescentes cuyas madres son casadas o en unión libre presentan las más altas puntuaciones para la dimensión académica $(\mathrm{M}=65.7 ; \mathrm{DT}=29.3)$, la dimensión familiar $(\mathrm{M}=62.8$; $\mathrm{DT}=32.8$;) y la dimensión física ( $\mathrm{M}=65.5$; $\mathrm{DT}=29.5)$. Además, los adolescentes cuyas madres son solteras, casadas, divorciadas y viudas muestran una puntuación por debajo del percentil 50 en la dimensión emocional, destacándose la puntuación más baja en las madres viudas $(\mathrm{M}=47.0 ; \mathrm{DT}=31.5)$ y en la dimensión social presenta una menor puntuación por debajo del percentil 50 en la madre divorciada o separada ( $M=41.7$; $D T=30.3)$.

En cuanto a la característica sociodemográfica, el nivel de estudios de las madres y las dimensiones del autoconcepto de los adolescentes, los resultados indican que los adolescentes cuencanos de madres con nivel de estudios: educación básica incompleta, educación básica completa, bachillerado, universidad y posgrado presentan puntuaciones sobre el percentil 50 en las dimensiones: académica, familiar, emocional y física. Sin embargo, se destaca que el nivel de estudios de posgrado de la madre manifiesta en los adolescentes mayores puntuaciones en la dimensión académica ( $\mathrm{M}=72.4 * ; \mathrm{DT}=24.3)$, familiar $(\mathrm{M}=68.2 ; \mathrm{DT}=31.0)$, emocional $\left(\mathrm{M}=60.6^{*} ; \mathrm{DT}=34.5\right)$, mientras que, la dimensión física puntuó más alto en aquellos adolescentes cuyas madres tenían educación básica completa $(\mathrm{M}=65.5 ; \mathrm{DT}=29.7)$. Adicionalmente los resultados reportan que la dimensión social presenta una puntuación por debajo del percentil 50 en madres con nivel de estudios de educación básica incompleta ( $\mathrm{M}=30.8$; DT=28.1).

Finalmente, al relacionar las dimensiones del autoconcepto con las variables sociodemográficas de las madres se encontró que la dimensión académica de los adolescentes cuencanos mostró una relación significativa entre el nivel de estudios (posgrado) de la madre puntuando más alto con el ( $\mathrm{M}=72.4^{*}$; $\left.\mathrm{DT}=24.3\right)$ con el estado civil (casada o unión libre) mostrando una mayor puntuación ( $\mathrm{M}=65.7 *$; $\mathrm{DT}=29.3)$. La dimensión social de los adolescentes se asocia con la jornada laboral de la madre, siendo 8 horas de trabajo la que presenta el promedio más alto $\left(\mathrm{M}=44.9^{*}\right.$; $\left.\mathrm{DT}=30.6\right)$. Respecto a la dimensión emocional al igual que la académica presenta una asociación con el nivel de estudios de la madre (posgrado), puntuando más alto ( $\mathrm{M}=60.6 *$; $\mathrm{DT}=34.5)$. La dimensión familiar y física no reporta ninguna relación con las características sociodemográficas de la madre.

Tabla 2. Autoconcepto de los adolescentes considerando las características de la madre.

\begin{tabular}{|c|c|c|c|c|c|c|c|c|c|c|c|}
\hline & & \multicolumn{2}{|c|}{ Académico } & \multicolumn{2}{|c|}{ Social } & \multicolumn{2}{|c|}{ Emocional } & \multicolumn{2}{|c|}{ Familiar } & \multicolumn{2}{|c|}{ Físico } \\
\hline & & $\mathrm{M}$ & DT & $\mathrm{M}$ & DT & $\mathrm{M}$ & DT & $M$ & DT & $M$ & DT \\
\hline \multirow{5}{*}{$\begin{array}{c}\text { Horas de } \\
\text { trabajo }\end{array}$} & $0-4$ & 65.0 & 29.5 & 43.0 & 31.9 & 48.4 & 32.4 & 63.3 & 33.3 & 63.5 & 30.2 \\
\hline & $4-7$ & 56.1 & 32.8 & 42.9 & 30.3 & 51.6 & 31.2 & 55.5 & 34.9 & 64.1 & 28.0 \\
\hline & 8 & 65.5 & 29.3 & $44.9^{*}$ & 30.6 & 52.2 & 32.2 & 61.5 & 32.8 & 64.9 & 29.7 \\
\hline & $9-12$ & 64.9 & 28.5 & 44.2 & 31.3 & 49.7 & 29.3 & 60.8 & 31.1 & 65.3 & 29.8 \\
\hline & $>12$ & 67.5 & 32.8 & 40.2 & 36.0 & 59.8 & 35.5 & 56.4 & 40.4 & 57.0 & 37.4 \\
\hline \multirow{4}{*}{$\begin{array}{l}\text { Estado } \\
\text { civil }\end{array}$} & Soltera & 58.1 & 31.4 & 43.3 & 33.0 & 53.5 & 32.1 & 62.5 & 31.1 & 58.9 & 31.6 \\
\hline & $\begin{array}{l}\text { Casada o } \\
\text { unión libre }\end{array}$ & $65.7 *$ & 29.3 & 44.7 & 31.3 & 50.1 & 31.5 & 62.8 & 32.8 & 65.5 & 29.5 \\
\hline & Divorciada o separada & 60.6 & 30.0 & 41.7 & 30.3 & 49.9 & 33.3 & 53.5 & 33.7 & 63.1 & 29.7 \\
\hline & Viuda & 60.6 & 30.7 & 53.2 & 34.5 & 47.0 & 31.5 & 59.3 & 37.2 & 64.1 & 35.2 \\
\hline \multirow{5}{*}{$\begin{array}{l}\text { Nivel de } \\
\text { estudios }\end{array}$} & Educación B. Incompleta & 49.6 & 31.4 & 30.8 & 28.1 & 50.9 & 36.8 & 57.3 & 35.3 & 56.3 & 36.4 \\
\hline & Educación B. completa & 60.0 & 30.0 & 42.8 & 30.5 & 47.2 & 30.9 & 57.8 & 32.9 & 65.6 & 29.7 \\
\hline & Bachillerato & 66.0 & 29.9 & 46.7 & 32.2 & 49.6 & 32.2 & 64.1 & 32.9 & 64.2 & 30.1 \\
\hline & Universidad/Técnico & 68.1 & 28.5 & 45.8 & 31.6 & 54.0 & 31.3 & 63.4 & 33.0 & 64.6 & 29.8 \\
\hline & Postgrado & $72.4^{*}$ & 24.3 & 37.7 & 31.6 & $60.6^{*}$ & 34.5 & 68.2 & 31.0 & 60.2 & 28.1 \\
\hline
\end{tabular}

Nota: * (p-valor<0.05). 


\section{Dimensiones del autoconcepto de los adolescentes con relación a las características sociodemográficas del padre.}

Los resultados de la relación entre las dimensiones del autoconcepto y las características sociodemográficas de los padres reportan lo siguiente: en cuanto a la jornada laboral se encontró que los adolescentes cuencanos con padres que trabajan 8 horas o más presentan puntuaciones por encima del percentil 50 en la dimensión académica $(\mathrm{M}=65.2 ; \quad \mathrm{DT}=29.9 ; \quad \mathrm{M}=64.8 ; \quad \mathrm{DT}=28.9 ; \quad \mathrm{M}=79.6$; $\mathrm{DT}=21.9) ; \quad$ familiar $\quad(\mathrm{M}=63.6 ; \quad \mathrm{DT}=31.8 ; \quad \mathrm{M}=61.9$; $\mathrm{DT}=33.7 ; \mathrm{M}=63.5 ; \mathrm{DT}=31.4)$, física $(\mathrm{M}=64.6$; $\mathrm{DT}=29.9$; $\mathrm{M}=63.7 ; \mathrm{DT}=30.3 \mathrm{M}=66.8 ; \mathrm{DE}=34.4)$, y emocional $(\mathrm{M}=62.7 ; \mathrm{DT}=29.7)$. Por otra parte, los adolescentes con padres que trabajan menos de 8 horas muestran una mayor puntuación por encima del percentil 50 en la dimensión académica $(\mathrm{M}=60.9 ; \mathrm{DT}=32.7 ; \mathrm{M}=66.6 ; \mathrm{DT}=26.2)$, familiar ( $\mathrm{M}=54.5 ; \mathrm{DT}=33.5 ; \mathrm{M}=59.1 ; \mathrm{DT}=34.9)$ y física $(\mathrm{M}=56.1 ; \mathrm{DT}=31.0 ; \mathrm{M}=65.7 ; \mathrm{DT}=28.5)$ y la dimensión emocional presenta una puntuación elevada ( $\mathrm{M}=61.0$; $\mathrm{DT}=29.1$ ) en el rango de 4 a 7 horas; mientras que la dimensión social se ubica por debajo del percentil 50. Respecto al cruce de la variable del estado civil de padre y las dimensiones del autoconcepto, los resultados informan que los adolescentes cuencanos cuyo estado civil del padre responde a estar soltero, casado o en unión libre, divorciado y viudo presentan puntuaciones sobre el percentil 50 en las dimensiones: académica, familiar y física. Se destaca que los padres viudos presentan las más altas puntuaciones para la dimensión académica $(\mathrm{M}=68.3$; $\mathrm{DT}=33.3$ ), los padres casados o en unión libre para la dimensión familiar ( $\left.\mathrm{M}=63.6^{*} ; \quad \mathrm{DT}=32.7\right)$ y física $(\mathrm{M}=65.7 ; \mathrm{DT}=29.5)$. Además, los adolescentes de aquellos padres que son casados, divorciados y viudos muestran una puntuación por debajo del percentil 50 en la dimensión emocional, destacándose la puntuación más baja en los padres viudos ( $M=43.3$; $D T=44.5)$, al igual que la dimensión social $(\mathrm{M}=20.0 ; \mathrm{DT}=15.5)$.

En cuanto al nivel de estudios de los padres y las dimensiones del autoconcepto de los adolescentes los resultados apuntan a que los adolescentes cuencanos de padres con nivel de estudios: educación básica incompleta, educación básica completa, bachillerado, universidad y posgrado presentan puntuaciones sobre el percentil 50 en las dimensiones: académica, emocional, familiar y física, se destaca que el nivel de estudios de posgrado presentan mayores puntuaciones en la dimensión académica $\left(\mathrm{M}=74.3^{*}\right.$; $\left.\mathrm{DT}=26.5\right)$, y emocional $\left(\mathrm{M}=57.1^{*} ; \mathrm{DT}=34.2\right)$. Sin embargo, la educación básica incompleta presenta una elevada puntuación en la dimensión familiar $(\mathrm{M}=71.8$; $\mathrm{DT}=27.0)$ y física $(\mathrm{M}=77.4$; $\mathrm{DT}=19.5$ ), adicionalmente los resultados reportan que la dimensión social presenta una puntuación por debajo del percentil 50 en padres con nivel de estudios de educación básica incompleta $(\mathrm{M}=34.4$; $\mathrm{DT}=38.3)$.

En la relación de las dimensiones del autoconcepto con las características sociodemográficas de los padres de los adolescentes cuencanos se encontraron relaciones significativas como se muestra a continuación: el nivel de estudios (posgrado) del padre se relaciona con la dimensión académica $\left(\mathrm{M}=74.3^{*}\right.$; $\left.\mathrm{DT}=26.5\right)$ y emocional $\left(\mathrm{M}=57.1^{*} ; \mathrm{DT}=34.2\right)$. Los datos evidencian que, a mayor nivel de estudios en los padres, se presenta un mayor autoconcepto ( $\rho$ académica $=.123^{* *} ; \quad \rho$ emocional $=$ $\left..133^{* *}\right)$. Además, se encontró una relación significativa del estado civil casado o unión libre del padre, con una alta puntación para la dimensión familiar (M=63.6*; $\mathrm{DT}=32.7)$, y física $(\mathrm{M}=65.7 *$; $\mathrm{DT}=29.5)$. Estos resultados están recogidos en la Tabla 3.

\section{DISCUSIÓN}

Los resultados reportan que de la muestra total la dimensión académica y física presenta idénticas puntuaciones, las mismas, son más elevadas que las otras dimensiones del autoconcepto. En cuanto a las diferencias del sexo de los adolescentes, son las mujeres las que presentan una mayor puntuación en la dimensión académica, física y emocional, en tanto que los hombres evidencian una puntuación mayor dimensión familiar. La puntuación alta de la dimensión académica en las mujeres es similar con lo hallado por Rodríguez y Ramos (2015), quienes encontraron que el autoconcepto académico elevado en las mujeres y que está fuertemente relacionado con el rendimiento académico. Así también, otro planteamiento señala indicios de que los adolescentes que se perciben con buen autoconcepto académico es el

Tabla 3. Autoconcepto de los adolescentes de acuerdo con las características del padre.

\begin{tabular}{clcccccccccc}
\hline & & \multicolumn{3}{c}{ Académico } & \multicolumn{3}{c}{ Social } & \multicolumn{3}{c}{ Emocional } & \multicolumn{3}{c}{ Familiar } & \multicolumn{2}{c}{ Físico } \\
\cline { 2 - 11 } & & M & DT & M & DT & M & DT & M & DT & M & DT \\
\hline \multirow{4}{*}{$\begin{array}{c}\text { Horas de } \\
\text { trabajo }\end{array}$} & $4-4$ & 66.6 & 26.2 & 44.7 & 31.4 & 45.9 & 34.0 & 59.1 & 34.9 & 65.7 & 28.5 \\
& $4-12$ & 60.9 & 32.7 & 43.5 & 28.8 & 61.0 & 29.1 & 54.5 & 33.5 & 56.1 & 31.0 \\
& $>12$ & 65.2 & 29.9 & 43.3 & 31.4 & 49.2 & 31.0 & 63.6 & 31.8 & 64.6 & 29.9 \\
& Soltero & 64.8 & 28.9 & 44.2 & 31.7 & 50.8 & 31.1 & 61.9 & 33.7 & 63.7 & 30.3 \\
& Casado o Unión libre & 69.6 & 21.9 & 42.5 & 36.3 & 62.7 & 29.7 & 63.5 & 31.4 & 66.8 & 34.4 \\
\hline \multirow{5}{*}{ Estado civil } & 62.9 & 30.3 & 39.5 & 31.9 & 55.5 & 31.1 & 48.0 & 31.5 & 48.2 & 29.6 \\
& Divorciado o separado & 58.9 & 29.6 & 43.9 & 31.6 & 49.9 & 31.4 & $63.6 *$ & 32.7 & 65.7 & 29.5 \\
& Viudo & 68.3 & 33.3 & 20.0 & 15.0 & 43.3 & 44.5 & 60.0 & 3.6 & 41.3 & 50.5 \\
\hline \multirow{5}{*}{ Nivel de } & Educación B. Incompleta & 67.8 & 33.0 & 34.4 & 38.3 & 38.2 & 36.2 & 71.8 & 27.0 & 77.4 & 19.5 \\
estudios & Educación B. Completa & 61.7 & 29.0 & 41.1 & 31.0 & 44.4 & 31.5 & 59.8 & 32.9 & 64.1 & 31.0 \\
& Bachillerato & 65.1 & 29.6 & 45.3 & 31.3 & 51.9 & 30.9 & 61.9 & 33.6 & 65.0 & 29.1 \\
& Universidad/Técnico & 67.6 & 29.0 & 46.0 & 3.9 & 53.2 & 29.9 & 63.3 & 32.4 & 62.2 & 29.8 \\
& Postgrado & $74.3 *$ & 26.5 & 46.1 & 30.1 & $57.1 *$ & 34.2 & 66.4 & 32.7 & 69.9 & 28.1 \\
\hline
\end{tabular}

Nota: * (p-valor<0.05) 
resultado de la retroalimentación realizada por los padres, pues esto favorece el desarrollo del autoconcepto académico (Álvarez et al., 2015). En esta dirección Fernández y Samaniego (2020), señalan que el autoconcepto académico depende también de otros factores como la situación social, económica y educativa de la familia. Este argumento apoya los objetivos de este estudio al considerar la mediación de otros factores en la variabilidad de las dimensiones del autoconcepto.

En cuanto a la dimensión física los resultados encontrados son similares a lo observado en estudios previos que reportan una puntuación alta para la dimensión física en grupos adolescentes españoles y cuencanos (Barrios et al.,2017; Fernández \& Samaniego, 2020; Rangel et al., 2017). Estos autores observan que la puntuación alta para la dimensión física podría estar relacionada con la perspectiva e importancia que las adolescentes le dan a su atractivo físico y lo consideran como un factor influyente al momento de establecer relaciones interpersonales. En este estudio los adolescentes que asisten al tercero año de bachillerato presentan mayores puntuaciones en la dimensión física, este resultado es similar en adolescentes españoles (Rangel et al., 2017; Reiga et al., 2012), no obstante, no es coincidente con la investigación de Cazalla \& Molero (2013) e Iniesta \& Mañas (2014) que indican que se produce una disminución del interés por lo físico mientras avanza la edad. Estos resultados en torno a la dimensión física muestran variabilidad en función de la escolaridad que cursan los adolescentes.

La dimensión social reporta puntuaciones ligeramente menores en relación con el punto medio del percentil y son las mujeres las que muestran el menor autoconcepto social de la muestra total. Estos datos son similares a lo hallado por García et al. (2011) quienes evidenciaron en el caso de las adolescentes que la dimensión social muestra la menor puntuación. Autores observan que cuando en la adolescencia se presenta un autoconcepto negativo se pierde el sentido de identidad social y se pierde la capacidad de socializar (Fuentes et al., 2011; Moreno et al., 2017).

Respecto a la relación de las dimensiones del autoconcepto con el tipo de sostenimiento de las instituciones educativas, se encontró un mejor autoconcepto académico y familiar en los adolescentes que asisten a las instituciones particulares. Estos resultados son similares al estudio de Suldo et al. (2011) donde reportan que el autoconcepto general se asocia de manera significativa con el autoconcepto académico de los estudiantes de las instituciones particulares, en tanto que los estudiantes de las instituciones públicas no presentaron asociación significativa entre estas variables. Otra razón que explica altas puntuaciones en la dimensión académica y familiar responde a que diversos entornos socioeconómicos, también generarían un impacto en el autoconcepto de los adolescentes, en dimensiones como la autopercepción corporal, el rendimiento académico y la autopercepción familiar y social (Cleric et al., 2020).

En cuanto a la relación de las dimensiones del autoconcepto con la tipología familiar, los datos muestran que los adolescentes que pertenecen a la familia nuclear presentan mayor puntuación del autoconcepto familiar en comparación a la familia reconstituida y la familia monoparental paterna. No es posible contrastar nuestros resultados ya que no se dispone de otros estudios que hayan relacionado estas variables, sin embargo, algunos autores señalan que los hijos pueden presentar un buen autoconcepto y ajuste psicosocial en cualquier estructura familiar, y se pueden mantener vínculos afectivos para favorecer el bienestar de los miembros de la familia (Oliva et al., 2014; Oudhof et al., 2018; Martínez et al., 2013; Viveros, 2016).

El segundo objetivo fue analizar las dimensiones del autoconcepto del adolescente relacionado con las características sociodemográficas de los progenitores según la jornada de trabajo, el estado civil y el nivel de estudios. Respecto a la relación del autoconcepto del adolescente con las características sociodemográficas de sus progenitores, la jornada laboral del padre no presenta ninguna relación con las dimensiones del autoconcepto; sin embargo, esta variable en el caso de la madre se relaciona con el autoconcepto social del adolescente. Nuestros resultados son similares con una investigación realizada por Cantón et al. (2010) en el cual las madres que trabajan más de 12 horas presentaron un valor menor en las dimensiones del autoconcepto social y emocional. Esto sugiere que la madre asume una mayor implicación con los adolescentes, lo que genera que estos se encuentran en una situación de abandono parcial, por lo que exteriorizan una imagen social disminuida. Situación que no ocurre cuando es el padre el que se ausenta más horas, debido a que generalmente son los padres los que fomentan la autonomía de los hijos (Cleric et al., 2020). Dos variables de los progenitores presentan una relación significativa: el estado civil (casado y unión libre) y el nivel de estudios (posgrado). Respecto al estado civil de los progenitores se encontró que el estado civil de las madres favorece el autoconcepto académico, en tanto que esta misma variable en los padres muestra una relación significativa con el autoconcepto familiar y físico del adolescente. .En cuanto a la dimensión emocional de los adolescentes hombres y mujeres, si bien, los resultados presentan puntuaciones por debajo del percentil 50 en la relación con madres y padres viudos, se observa una relación significativa con la familia nuclear. Nuestros resultados no los podemos discutir con otros estudios porque la investigación interesada en la relación entre el estado civil de los progenitores y el autoconcepto de los adolescentes es escasa, sin embargo, diferentes investigaciones manifiestan el importante efecto de la familia sobre el autoconcepto, siendo los sujetos con niveles elevados de autoconcepto y adaptación personal aquellos que perciben su ambiente familiar más cohesionado y poco conflictivo (González-Pienda et al., 2002; Pichardo \& Amezcua, 2001; Putnick et al., 2008; De La Torre et al., 2013). De este planteamiento podemos inferir que los progenitores casados o en unión libre que presenten relaciones cohesionadas y funcionales en la crianza de los hijos podrían favorecer un buen desarrollo del autoconcepto y ajuste social de su progenie.

Finalmente, respecto al nivel de estudios de los progenitores relacionado con las diversas dimensiones del autoconcepto, los resultados muestran que aquellos adolescentes cuyos progenitores poseen estudios de posgrado presentaron un nivel de autoconcepto mayor en todas sus dimensiones, con la particularidad que se presenta una relación significativa entre los estudios de posgrado tanto de madre como de padre con el autoconcepto académico y emocional. Nuestros resultados concuerdan con el estudio de Soto et al. (2018), quienes tras un análisis concluyeron que el estudiante cuyo progenitor posee estudios superiores tiene valores más elevados de autoconcepto general, en comparación con adolescentes cuyos padres presentan estudios medios y básicos. Varios autores mencionan que un alto nivel de 
estudios de los progenitores conduce a que estos privilegien prácticas de apoyo a la autonomía para desarrollar en los hijos un buen autoajuste que conduce a un buen autoconcepto académico y rendimiento escolar, como también, favorece a un buen autoconcepto emocional, social y familiar (Cleric et al., 2020; Gasa Molepo \& Lethole, 2018; Morsy \& Rothstein, 2015; Rodríguez- Fernández et al., 2019). En este sentido el nivel educativo de padres y madres es la variable con mayor poder predictivo para el desarrollo de los menores y adolescentes (Montroy et al., 2016; Neidhöfer et al., 2018).

\section{CONCLUSIONES}

El desarrollo de este estudio nos permite concluir en términos generales que los adolescentes cuencanos presentan puntuaciones por encima del percentil 50, lo cual representa un autoconcepto estable en la dimensión académica y física; seguidas por la dimensión familiar y emocional; mientras que, la dimensión social reporta una puntuación ligeramente por debajo del punto de corte.

En lo referente a la relación entre las dimensiones del autoconcepto con las características sociodemográficas del adolescente y de los progenitores, los resultados muestran relaciones significativas: entre la dimensión académica y emocional con el nivel de estudios de posgrado del padre y la madre, el estado civil casadas o en unión libre de la madre y con las instituciones particulares a las que asisten los adolescentes; la dimensión familiar se relacionó con las instituciones particulares, la familia nuclear y los padres estén casados o en unión libre; la dimensión física, con la escolaridad de los adolescentes y el estado civil del padre; y la dimensión social presentó una relación significativa con la jornada laboral de la madre. Se destaca que la dimensión emocional de los adolescentes hombres y mujeres en la relación con las características sociodemográficas de los progenitores presenta puntuaciones por debajo del percentil 50 tanto en madres y padres viudos. Los hallazgos encontrados en el estudio permiten confirmar las características del autoconcepto, la capacidad de permanecer estable y cambiante al mismo tiempo. Es así, que la identificación de las dimensiones generales del autoconcepto del adolescente cuencano responde a la estabilidad y la variabilidad de las dimensiones del autoconcepto se explica por los factores dependientes del contexto como son las características sociodemográficas de los adolescentes y de sus progenitores.

Esta investigación presenta algunas limitaciones que es preciso señalar: en primer lugar, la revisión del estado de arte del autoconcepto en función de sexo, sostenimiento de las instituciones educativas, escolaridad, jornada laboral, estado civil y nivel de estudios donde se evidencia las escasas investigaciones interesadas en la relación de estas variables que permiten una adecuada contrastación de los resultados encontrados en este estudio, y, en segundo lugar, las variables de estudio no permitieron establecer el efecto del autoconcepto en comportamientos particulares de los adolescentes. En futuras investigaciones, a manera de ejemplo, se podría relacionar el autoconcepto académico con el rendimiento académico de los estudiantes. El estudio realizado tiene implicaciones educativas y contribuye de manera importante al enriquecimiento de programas de intervención psicoeducativo y social en ámbito familiar para que los progenitores refuercen del autoconcepto en la adolescencia, prestando atención a la dimensión emocional y social del adolescente cuencano.

\section{REFERENCIAS}

Álvarez, A., Suárez, N., Tuero, E., Núñez, J., Valle, A., \& Regueiro, B. (2015). Implicación familiar, autoconcepto del adolescente y rendimiento académico. European Journal of Investigation in Health, Psychology and Education, 5(3), 293-311.

Barrios, D., Gómez, M.A., \& Barriopedro, M.I. (2017). Análisis del autoconcepto físico en estudiantes de enseñanza física. Revista de la Psicología del Deporte. 26(2), 45-53.

Cantón, I., Valle, R., Arias, A., Baelo, R., \& Cañón, R. (2010). Retos educativos en la sociedad del conocimiento. Union Europea.

Castaño, M., Sánchez, M. P., \& Viveros, E. F. (2020). Los roles en las dinámicas familiares de las familias homoparentales. Revista Latinoamericana de Estudios de Familia, 12(2), 153-174. https://doi.org/10.17151/rlef.2020.12.2.9.

Cazalla, N., \& Molero, D. (2013). Revisión teórica sobre el autoconcepto y su importancia en la adolescencia. Revista Eléctronica de Investigación y Docencia, 10, 43-64.

Cleric, G., Elgie, Á., Gago, L., \& García, M. (2020). La contribución del entorno socioeconómico al autoconcepto y percepción infantil de las pautas parentales de crianza. Revista de Psicología y Educación, 15(1), 87-97.

De La Torre, M.J., Cruz, Casanova, P.F, Villa, M., \& Cerezo, M.T. (2013). Consistencia e inconsistencia parental: relaciones con la conducta agresiva y satisfacción vital de los adolescentes. European Journal of Education and Psychology, 6(2), 135-149.

Delgado, B., Inglés, C. J., \& García-Fernández, J. M. (2013). Social anxiety and self-concept in adolescence. Revista de Psicodidáctica, 18, 179-194. https://doi.org/10.1387/RevPsicodidact.6411

Derdikman-Eiron, R., Indredavik, M. S., Bratberg, G. H., Taraldsen, G., Bakken, I. J., \& Colton, M. (2011). Gender differences in subjective well-being, selfesteem and psychosocial functioning in adolescents with symptoms of anxiety and depression: Findings from the Nord-Trøndelag health study. Scandinavian Journal of Psychology, 52, 261-267.

Erikson H., E. (1992). Identidad, juventud y crisis. Madrid: Taurus

Esnaola, I., Goñi, A., \& Madariaga, J. M. (2008). El autoconcepto: perspectivas de investigación. Revista de Psicodidáctica, 13(1), 179-194.

Esnaola, I., Rodríguez, A., \& Goñi, E. (2011). Propiedades psicométricas del cuestionario de Autoconcepto AF5. Anales de Psicología, 27(1), 109117.

Fernández Ortiz, D. S., \& Samaniego Reinoso, M. M. (2020). Influencia del control psicológico parental en 
el desarrollo del autoconcepto en adolescentes de tres unidades educativas fiscales en Cuenca en el periodo 2019-2020 Tesis de Grado. Universidad de Cuenca. http://dspace.ucuenca.edu.ec/handle/123456789/33996

Fuentes, M. C., García, F., Gracia, E., \& Lila, M. (2011). Autoconcepto y consumo de sustancias en la adolescencia. Adicciones, 23, 237-248.

Fuentes, M. García, F., Gracia, E., Alarcón, A. (2015). Los estilos parentales de socialización y el ajuste psicológico. Un estudio con adolescentes españoles. Revista de Psicodidáctica, 20(1), 117-138. https://doi.org/10.1387/RevPsicodidact.10876

Garaigordobil, M., \& Durá, A. (2006). Relaciones del autoconcepto y la autoestima con la sociabilidad, estabilidad emocional y responsabilidad en adolescentes de 14 a 17 años. Análisis y Modificación de Conducta, 32(141), 37-64.

García, J. (2002). Prácticas educativas familiares y autoconcepto. Estudio con niños y niña de 3, 4, y 5 años. Universidad de Valladolid. Departamento de Psicología. Tesis doctoral.

García, F., \& Musitu, G. (2014). AF5: Autoconcepto Forma 5. Madrid: Tea Ediciones.

García, F., Martínez, I., Balluerka, N., Cruise, E., García, O., \& Sierra, E. (2018). Validation of the Five-Factor Self-Concept Questionnaire AF5 in Brazil: Testing factor structure and measurement invariance across language (Brazilian and Spanish), gender, and age. Frontiers in Psychology, 9, 1-13.

García, J., \& Román Sánchez, J. (2005). Prácticas educativas familiares y autoestima. Psicothema, 17(1), 76-82.

Gasa, V., Pitsoane, E., Molepo, L., \& Lethole, P. (2018). The effect of families' socioeconomic status on the self-concept development of learners. Early Child Development and Care.

González-Pienda, J.A., Núñez Pérez, J.C., Álvarez, L., González-Pumariega, S., Roces, C., González, P., Muñiz, R., \& Bernardo, A. (2002). Inducción parental a la autorregulación, autoconcepto y rendimiento académico. Psicothema, 14, 853-860.

Goñi, A., \& Fernández, E. (2008). Educational support for enhancing physical self-concept. Problems of Education in the 21st Century, 6, 30-41.

Heatherton, T. F., \& Wyland, C. L. (2003). Assessing self-esteem. In S. J. Lopez \& C. R. Snyder (Eds.), Positive psychological assessment: Handbook of models and measures (pp. 219-233). Washington DC: American Psychological Association.

Inda-Caro, M., Rodríguez-Menéndez, C., FernándezGarcía, C., \& Viñuela-Hernández M (2020). Rol de las metas parentales orientadas al logro de la tarea en el autoconcepto de adolescentes universitarios. Revista Iberoamericana de Diagnóstico y Evaluación, 56(3), 143-158. https://doi.org/10.21865/RIDEP56.3.11

Iniesta, A., \& Mañas, C. (2014). Autococncepto y rendimiento académico en adolescentes. International Journal of Developmental and Educational Psychology, 2(1), 555-564.

Martínez, M., Estévez, E., \& Cándido, I. (2013). Diversidad familiar y ajuste psicosocial en la sociedad actual. Revista Psicologia.com,17(6).

Mérida, R., Serrano, A., \& Tabernero, C. (2015). Diseño y validación de un cuestionario para la evaluación de la autoestima en la infancia. Revista de Investigación Educativa, 33, 149-162. http://dx.doi.org/10.6018/rie.33.1.182391

Mistry, R., Stevens, G., Sareen, H., De Vogli, R., \&Halfo, N. (2007). Parenting-related stressors and selfreported mental health of mothers with young children. American Journal of PublicHealth, 97 (7), 1261-1268.

Montroy, J. J., Bowles, R. P., Skibbe, L.E., McClelland, M. M., \& Morrison, F. J. (2016). The development of self-regulation across early childhood. Developmental Psychology, 52(11), 1744. https://doi.org/10.1037/dev0000159

Moreno, J. A., Moreno, R., \& Cervelló, E. (2017). Relación del autoconcepto físico con las conductas de consumo de alcohol y tabaco en adolescentes. Adicciones, 21(2), 147-154.

Morsy, L., \& Rothstein, R. (2015). Five Social Disadvantages that depress student performance: Why schools alone can't close achievement gaps. Report. Economic Policy Institute.

Neidhöfer, G., Serrano, J., \& Gasparini, L. (2018). Educational inequality and intergenerational mobility in Latin America: A new database. Journal of Development Economics, 134, 329-349. https://doi.org/10.1016/j.jdeveco.2018.05.016

Oliva, A., Arranz, E., \& Parra, A. (2014). "Family structure and child adjustment in Spain". Journal of Child and Family Studies, 23(1), 10-19.

Oudhof, H., Mercado, A., \& Robles, E. (2018). Cultura, diversidad familiar y su efecto en la crianza de los hijos. Estudios sobre las culturas contemporáneas, 14(48), 1-19.

Pichardo, M. C., \& Amezcua, J. A. (2001). Importancia del autoconcepto y el clima familiar en la adaptación personal. Revista Galego-Portuguesa de Psicoloxía e Educación, 7, 181-191.

Putnick, D. L., Bornstein, M. H., Hendricks, C., Painter, K. M., Suwalsky, J. T. D., \& Collins, W. A. (2008). Parenting stress, perceived parenting behaviors, and adolescent self-concept in European American Families. Journal of Family Psychology, 22, 752-762.

Rangel, Y. S., Mayorga, D., Peinado, J. E., \& Barrón, J. C. (2017). Actividad física, autoconcepto físico y bienestar psicológico en adolescentes universitarias mexicanas. Revista de la Psicología del Deporte, 26(2), 61-69.

Reigal, R., Videra, A., Parra, J. L., \& Juárez, R. (2012). Actividad físico deportiva, autoconcepto físico y bienestar psicológico en la adolescencia. Revista Retos, nuevas tendencias en Educación Física, Deportes y Recreación, 22, 19-23.

Rodrigo, M., \& Palacios, J. (1998). La familia como contexto y la familia en contexto. En M. J. Rodrigo \& J. Palacios (Coords.) Familia y desarrollo humano (pp.25-67). Madrid: Alianza.

Rodríguez-Fernández, A., \& Ramos-Díaz, E. (2015). Autoconcepto y ajuste emocional. En A. FernándezZabala, \& L. Revuelta (Eds). Ajuste personal y social. Investigación psicoeducativa (pp. 91-102).

Ros, I., \& Zuazagoitia, A. (2015). Implicación escolar y autoconcepto. En A. Fernández-Zabala y L. Revuelta (Eds), Ajuste personal y social. Investigación 
psicoeducativa, 119-130. Donostia: EREIN.

Schunk, D. H. (2012). Teorías del Aprendizaje: Una perspectiva educativa. (6ta ed.) Mexico: Pearson.

Shavelson, J., Hubner, J. J., \& Stanton, G. C. (1976). Self-concept: Validation of construct interpretations. Review of Educational Research, 46, 407-442. https://doi.org/10.3102/00346543046003407

Soto, M., Cuña, I., Guetiérrez, M., \& Barreira, Á. (2018). Nivel educativo de los progenitores como factor mediado del estrés académico. Fundación Educación Médica, 21(1), 23-29.

Suldo, S. M., Huebner, E. S., Savage, J., \& Thalji, A. (2011). Promoting subjective wellbeing. En M. A.
Bray, T. J. Kehle, M. A. Bray y T. J. Kehle (Eds.), The Oxford handbook of school psychology (pp. 504-522). New York, NY: Oxford University Press.

Tomyn, A. J., \& Cummins, R. A. (2011). The subjective wellbeing of high-school students: Validating the personal wellbeing index-school children. Social Indicators Research, 101, 405-418. http://dx.doi.org/10.1007/s11205-010-9668-6

Viveros, E. (2016). La condición de o familiar: entre el parentesco, la afinidad y el lazo social. Revista Virtual Universidad Católica del Norte, 48, 228-238. 